\section{Signal transmitter}

Proc. Natl Acad. Sci. USA 114, E6703-E6709 (2017)

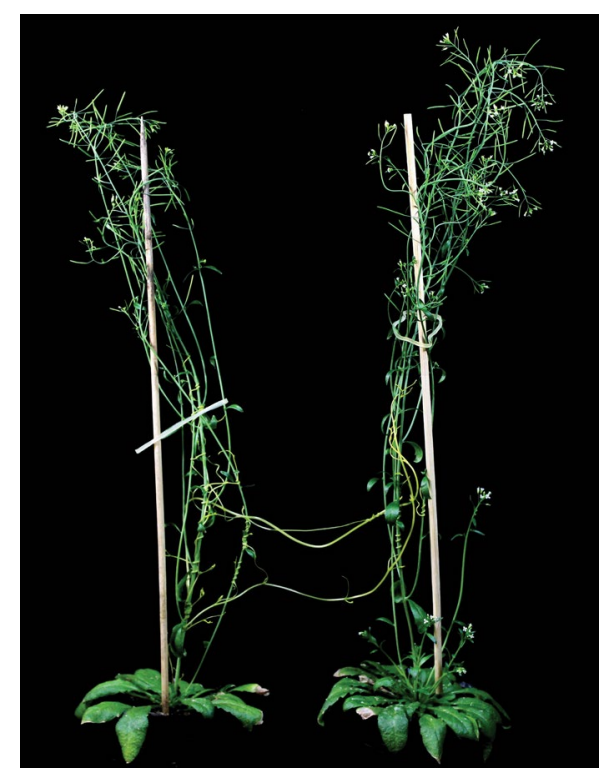

Credit: Jingxiong Zhang, Kunming Institute of Botany, Chinese Academy of Sciences

Dodders parasitize host plants by penetrating their haustoria organ into the host vascular system. One or more dodder plants often parasitize multiple host plants, forming connected plant clusters. Fusion to the host's vascular system not only allows dodders to extract water and nutrients, but also results in the transport of metabolites, proteins and mRNAs from the host into dodders. However, it remains unknown whether dodders can facilitate the transfer of ecologically meaningful signals among hosts in the connected plant clusters. Christian Hettenhausen, from the Kunming Institute of Botany, Chinese Academy of Sciences, and colleagues now experimentally show that dodder transfers herbivory-induced signals among host plants.

The researchers established a soybean plant cluster connected by dodders. After infestation with caterpillar, the leaves of both the attacked and undamaged plants showed dramatic transcriptome reprogramming, which did not happen for the undamaged plant if not connected by dodders. Meanwhile, both plants showed upregulation of anti-insect proteins and biosynthetic genes of monoterpenes, chemicals known to attract insect predators. Consistently, the undamaged plants gained higher insect resistance in insect feeding experiments, suggesting that dodders mediate inter-plant signalling and induce a defence response in the undamaged host.
Similar inter-plant signalling also occurred among heterospecific host plants in the Arabidopsis-dodder-tobacco and soybean-dodder-tomato systems, suggesting conservation of the interplant signalling. Replacing the wild-type Arabidopsis with a mutant impaired for jasmonic acid biosynthesis failed to prime the connected tobacco plants against insect attack. So the inter-plant signalling involves the jasmonic acid pathway.

The systemic signalling occurred rapidly, as the transcriptional response started in undamaged plants 30-45 minutes after the wounding. Using multi-plant clusters, the researchers showed that inter-plant signalling could travel across multiple host plants, reaching a distance of at least $100 \mathrm{~cm}$.

Identifying the underlying mobile molecules that mediate the transfer of the herbivory-induced signals among plants remains an intriguing task.

Jun Lyu

Published online: 28 August 2017

DOI: 10.1038/s41477-017-0015-7 